\title{
The citrus fruit-derived flavanone glycoside narirutin is a novel potent inhibitor of organic anion-transporting polypeptides
}

Tokio Morita ${ }^{1}$, Takeshi Akiyoshi ${ }^{1}$, Ryo Sato ${ }^{1}$, Yoshinori Uekusa ${ }^{1}$, Kazuhiro Katayama ${ }^{2}$, Kodai Yajima $^{1}$, Ayuko Imaoka ${ }^{1}$, Yoshikazu Sugimoto ${ }^{1}$, Fumiyuki Kiuchi $^{1}$, Hisakazu Ohtani ${ }^{1}$

${ }^{1}$ Graduate School of Pharmaceutical Sciences, Keio University, 1-5-30, Shibakoen, Minato-ku, Tokyo, 105-8512, Japan

${ }^{2}$ School of Pharmacy, Nihon University, 7-7-1 Narashinodai, Funabashi, Chiba, 274-8555, Japan

\section{Supporting Information}

Figure Cytotoxicity of a crude fraction separated from grapefruit juice 2

Table S1 Comparison of the observed and calculated residual uptake activities of estrone 3-sulfate in the presence of grapefruit juice or two glycosides 3 

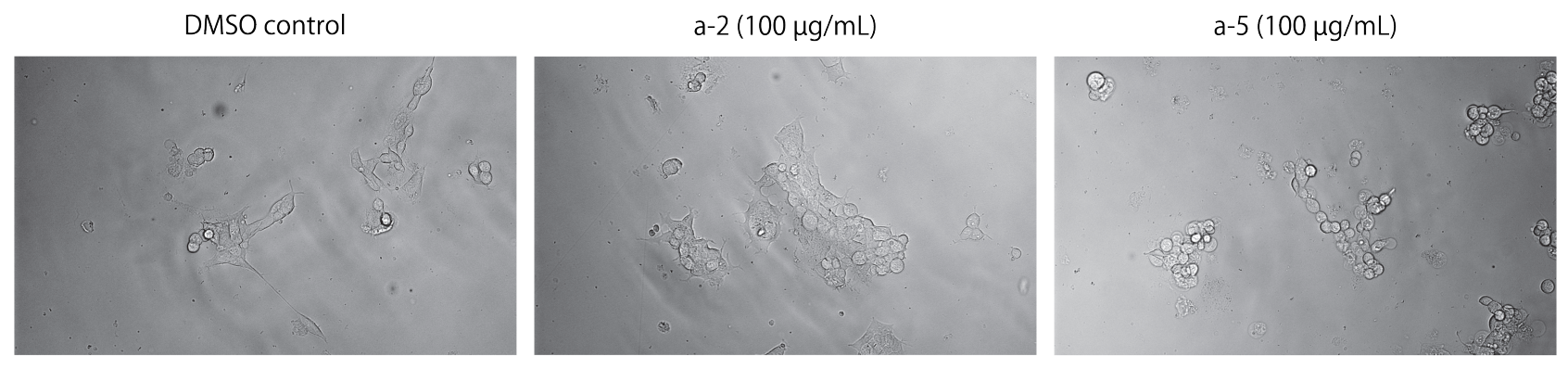

$100 \mu \mathrm{m}$

Figure Cytotoxicity of a crude fraction separated from grapefruit juice

The morphologies of HEK293-OATP2B1 cells that had been exposed to $100 \mu \mathrm{g} / \mathrm{mL}$ of the a-2 or a-5 fraction, which were derived from the methanol fraction separated from grapefruit juice, for $1 \mathrm{hr}$ are shown. Each fraction was dissolved in DMSO and then diluted to produce a final DMSO concentration of $0.1 \%$. Compared with the cells treated with the a- 2 fraction, those treated with the a-5 fraction at a concentration of $100 \mu \mathrm{g} / \mathrm{mL}$ were impaired. 
Table S1 Comparison of the observed and calculated residual uptake activities of estrone 3-sulfate in the presence of grapefruit juice or two glycosides

\begin{tabular}{|c|c|c|c|c|c|c|}
\hline & \multicolumn{3}{|c|}{$1 \%$ juice } & \multicolumn{3}{|c|}{$5 \%$ juice } \\
\hline & \multicolumn{2}{|c|}{ observed $^{\mathrm{a}}$} & \multirow[t]{2}{*}{ calculated } & \multicolumn{2}{|c|}{ observed $^{\mathrm{a}}$} & \multirow[t]{2}{*}{ calculated } \\
\hline & GFJ & $\mathrm{NR}+\mathrm{NN}^{\mathrm{b}}$ & & GFJ & $\mathrm{NR}+\mathrm{NN}^{\mathrm{b}}$ & \\
\hline OATP1A2 & $72 \pm 8.6 \%$ & $84 \pm 17 \%$ & $69 \%$ & $29 \pm 8.1 \%$ & $74 \pm 11 \%$ & $36 \%$ \\
\hline OATP2B1 & $60 \pm 5.8 \%$ & $88 \pm 10 \%$ & $72 \%$ & $39 \pm 17 \%$ & $51 \pm 10 \%$ & $42 \%$ \\
\hline
\end{tabular}

a, Observed uptake activity was obtained from Figure $1 \mathrm{C}$ and D.

b, Mixture of narirutin and naringin. 